\title{
Role of endophytic fungi on arsenic uptake and tolerance in
}

\section{Schizachyrium scoparium}

Cherie L. DeVore ${ }^{1 *}$, Eliane El Hayek ${ }^{1}$, Taylor Busch ${ }^{1}$,

Benson Long ${ }^{1}$, Paul Owen-Smith ${ }^{2}$, Jennifer Rudgers ${ }^{2}$,

Abdul Mehdi Ali ${ }^{3}$, Carlyle Ducheneaux ${ }^{4}$, José M.

\section{Cerrato $^{1}$}

${ }^{1} 1$ Univ of New Mexico, MSC 03 2040, cdevore@unm.edu

Abstract: We integrated aqueous chemistry,

multiplex Illumina sequencing, microscopy, and spectroscopy techniques to evaluate arsenic (As) uptake and tolerance in hydroponically grown Schizachyrium scoparium inoculated with endophytic fungi. Preliminary data detected $1 \mathrm{mg} \mathrm{kg}^{-1}$ accumulated in roots. In the absence of endophytes, $40 \mathrm{mg}$ $\mathrm{kg}^{-1}$ accumulated in roots of laboratory grown grass exposed to As. Dark septate endophytes (DSE) are a type of endophyte that are known to colonize the root tissues of plants and provide multiple benefits to warm and cool season grasses, such as Schizachyrium scoparium. Results from our study show that sterilized seedlings inoculated with DSE species enhanced plant biomass compared to non-inoculated plants. The objective of this study was to determine the effect of dark septate endophytes (DSE) on As uptake. we will do multiplexing with Illumina sequencing and ITS4fun primers for analyses of the fungal communities in our root samples. 